\title{
SHORT REPORT: DIFFERENCES IN DIHYDROFOLATE REDUCTASE BUT NOT DIHYDROPTEROATE SYNTHASE ALLELES IN PLASMODIUM FALCIPARUM ISOLATES FROM GEOGRAPHICALLY DISTINCT AREAS IN MALAYSIA
}

\author{
JANET COX-SINGH, ROBAIZA ZAKARIA, MOHD SHUKRI ABDULLAH, HASAN ABDUL RAHMAN, \\ SETHU NAGAPPAN, AND BALBIR SINGH \\ Faculty of Medicine and Health Sciences, University Malaysia Sarawak, Sarawak, Malaysia; School of Medical Sciences, \\ University Sains Malaysia, Kubang Kerian, Kelantan, Malaysia; State Department of Health, Kota Kinabalu, \\ Sabah, Malaysia; General Hospital, Tawau, Sabah, Malaysia
}

\begin{abstract}
Dihydropteroate synthase (dhps) and dihydrofolate reductase (dhfr) alleles were typed in 67 Malaysian Plasmodium falciparum isolates. The isolates were collected from two geographically distinct locations: 51 from Sabah, Malaysian Borneo, where sulfadoxine/pyrimethamine (SDX/PYR) is used to treat uncomplicated malaria and 16 from Peninsular Malaysia where in vivo resistance to SDX/PYR has been reported. A total of seven dhps alleles were identified with no significant difference in allele frequency between the 2 populations. Two of the dhps alleles described here have not been previously reported. Four $d h f r$ alleles were detected in 67 P. falciparum isolates. Eightyseven percent of the isolates from the Peninsula, where clinical SDX/PYR failure has been reported, had $d h f r$ alleles with triple point mutations while all of the isolates from Sabah had $d h f r$ alleles with 2 or less point mutations. The difference in $d h f r$ allele frequency between the two populations was highly significant. There was no correlation between in vitro PYR response and accumulation of $d h f r$ point mutations.
\end{abstract}

Fansidar, sulfadoxine/pyrimethamine (SDX/PYR) antimalarial combination therapy, has been used to treat uncomplicated Plasmodium falciparum malaria in areas where there is chloroquine resistance. Sulfadoxine and pyrimethamine inhibit different enzymes in the folate biosynthetic pathway, dihydropteroate synthase (dhps) and dihydrofolate reductase $(d h f r)$ respectively, and act synergistically when used in combination. ${ }^{1}$ In endemic areas where SDX/PYR treatment has replaced chloroquine, clinical drug failure has been reported at various intervals following SDX/PYR introduction. ${ }^{2}$ Clinical performance of antimalarial treatment may be measured by in vivo drug response assays and estimated by in vitro drug sensitivity assays. It is difficult to measure the response to the SDX/PYR combination in vitro $^{3,4}$ and the in vitro response to the PYR moiety alone has been used to indicate potential resistance to the SDX/PYR combination therapy. ${ }^{5}$

Non-silent point mutations on the dhps and $d h f r$ genes have been reported following the introduction of SDX/PYR therapy. ${ }^{6-8}$ The $d h f r$ mutations have been shown to alter the PYR-dhfr association constant reducing the efficiency of the drug in vitro. ${ }^{9}$ In addition there appears to be a sequential progression in the number of mutations on the $d h f r$ gene that correlate with an increased incidence of clinical drug failure and reduced response in vitro. The point mutation at $d h f r$ position 108 is detected first, followed by point mutations at positions 50 and/or 59 and finally position 164.,210,11 For dhps, mutations have also been detected following SDX/ PYR therapy. 2,6,12 The evidence for sequential dhps mutational events is not as strong but the point mutation at position 437 is thought to be the first event and is associated with a decreased response to SDX. ${ }^{6,7}$ SDX/PYR has been in use for more than 20 years in Malaysia. The epidemiology of malaria in Malaysia, shows a contrast between Peninsular Malaysia on mainland Asia and the states of Sabah and Sarawak on the island of Borneo. Malaria is largely under control in Peninsular Malaysia despite the fact that clinical resistance to SDX/PYR has been reported in various locations. ${ }^{13}$ However, in Sabah (Malaysian Borneo), SDX/PYR remains the drug of choice for treating uncomplicated falciparum malaria. That the drug has been used for such a prolonged period in Sabah without the widespread emergence of clinical failure is unusual given that resistance to SDX/PYR has emerged rapidly in Southeast Asian (including Peninsular Malaysia), South American, and African countries..$^{2,14,15}$ In this study, point mutations on the dhps and $d h f r$ genes were typed in P. falciparum isolates collected from Kelantan, Peninsular Malaysia where clinical resistance to SDX/PYR has been reported ${ }^{13}$ and from Sabah where SDX/PYR remains the recommended drug of choice for uncomplicated malaria. Dhps and $d h f r$ alleles were mapped and the potential of molecular typing as a marker for emergent drug resistance is discussed.

\section{MATERIALS AND METHODS}

The study was conducted on P. falciparum isolates collected from patients at Lahad Datu Hospital, Kunak Health Center, and Tawau General Hospital, in Southern Sabah, Malaysian Borneo during December 1996, May 1997, and December 1997. Patients with slide-positive $P$. falciparum single infections were recruited into the study following a full explanation of the study protocol and having obtained patient or parent/guardian consent.

Three $\mathrm{ml}$ venous blood were collected of which $0.5 \mathrm{ml}$ was placed into heparin tubes for World Health Organization (WHO) in vitro drug assays and the remainder into EDTA blood collection tubes. The EDTA blood was used for making thick and thin blood films, $10 \times 20 \mu$ l bloods spots on filter paper and the remainder cryopreserved. In addition, blood-spot samples from Peninsula Malaysia were included in the molecular typing study. These samples were collected between August 1994 and August 1995 from two district hospitals, namely Tanah Merah and Kuala Krai in the state of Kelantan situated on the Eastern Malaysian/Thai border. The study protocol was approved by the Ethics Committee of the Ministry of Health Malaysia.

For mutation detection, DNA was extracted from $20 \mu l$ of 